(C) Hosny G.A., Ahmed A.-S. A.-A., 2021

DOI 10.18019/1028-4427-2021-27-3-351-356

\title{
Is arthroplasty inevitable after Ilizarov hip reconstruction of unstable hip joints in adolescents and young adults? Long-Term Evaluation of 136 Cases
}

\author{
G.A. Hosny, A.-S. A.-A. Ahmed \\ Benha University, Faculty of Medicine, Egypt
}

\begin{abstract}
Introduction Whereas hip joint destroying trauma and diseases are difficult situations, the problem is more complex when it is complicated by hip instability. This could be a sequel of several hip affections such as trauma, septic or tuberculous arthritis, neglected developmental dysplasia of the hip, postoperative conditions, and neurologic pathologies (cerebral palsy, myelomeningocele, poliomyelitis). Purpose The purpose of this study is to evaluate long-term radiographic and clinical outcomes of the Ilizarov hip reconstruction for the treatment of painful and unstable hips in adolescents and young adults. Materials and methods The study included 136 patients with an average age of 18.3 years (range, 6 to 34 years); 75 patients were males $(55.1 \%)$ and 61 females (44.9\%). The primary causes of the hip instability were untreated or unsuccessfully treated cases of septic arthritis (40 cases; $29.4 \%$ ), congenital hip dislocation (28 cases; $20.6 \%$ ), paralytic hip dislocation ( 36 cases; $26.5 \%$ ), proximal femoral focal deficiency (14 cases; $10.3 \%$ ), neglected fracture of the femoral neck (10 cases; $7.4 \%$ ), osteoarthritis ( 6 cases; $4.4 \%$ ), and tuberculous hip arthritis ( 2 cases; $1.5 \%$ ). The intervention consisted in the performance of two osteotomies (proximal and distal) of the femur with pelvic support and placement of the Ilizarov apparatus of a specific assembly. Results The external fixation period ranged from 4 to 12 months (6.5 months on average). Patients were followed up for an average of 17.4 years (range, 5 to 27 years). Multiple clinical parameters at final follow-ups showed significant improvement, including pain relief, pain-free walking distance, lameness, hip flexion and abduction, hip contracture, and lumbar lordosis. Functionally, the mean Harris Hip Score improved with a statistically significant difference from 48 points (range, 35-65) before surgery to 83 points (range 70-90) after surgery. The pain disappeared in all patients, with the exception of six cases of pain in the early postoperative period. In all cases, supportive walking aids were no longer necessary, with the exception of two cases of persistent pain by physical activities. Walking ability and painless walking distance improved in all patients from an average of $35 \mathrm{~m}$ (range, 10 to $50 \mathrm{~m}$ ) before surgery to $1,150 \mathrm{~m}$ (range, 1,000 to $1,500 \mathrm{~m}$ ) after surgery, showing significant difference. Conclusion Ilizarov pelvic support osteotomy provided a multi-purpose solution to the complex challenging problem of hip instability in adolescents and young adults with variable primary etiologies. The improvements in the hip motion, mechanical axis, and correction of limb-length discrepancy lead to good functional outcomes over a long-term follow-up. This treatment modality might avoid or postpone the need for total hip arthroplasty for several years.

Keywords: adolescents, young people, hip joint, instability, reconstruction, supporting osteotomy, Ilizarov apparatus
\end{abstract}

\section{INTRODUCTION}

Whereas hip joint destroying trauma and diseases are difficult situations, the problem is more complex when it is complicated by hip instability. This could be a sequel of several hip affections such as trauma, septic or tuberculous arthritis, neglected developmental dysplasia of hip, Girdlestone operation, and neurologic pathologies (cerebral palsy, myelomeningocele, poliomyelitis). Hip dysfunction in adolescents and young adults is a complex condition which could result in serious consequences severely affecting their quality of life if not treated correctly and timely $[1,2]$.

This challenging problem has several factors contributing to complexity of the situation. The unstable hip is usually accompanied with proximal femoral bone loss, shortening, gait abnormalities, and pain [3]. Moreover, the unstable fulcrum caused by proximal femoral migration occurs on loading which consequently weakens the abductor gluteal muscles, shortens the lever arm, and eventually leads to Trendelenberg gait. Initially limping is painless but becomes painful and reduces walking tolerance. This migration of the femur results in adduction contracture with posterior displacement of the femoral head. Secondarily, the center of gravity will be anterior to the head of femur with ensuing anterior pelvic rotation and increased anterior tilt.
In addition to hip flexion contracture, this pelvic rotation increases the compensatory lumbar lordosis and contributes to low back pain $[3,4]$.

This established complexity results in limited options for treatment including arthrodesis, total hip replacement (THR), and pelvic support osteotomy. The goal of treatment for these unstable hip joints in adolescents and young adults is to alleviate pain, improve joint range of motion (ROM), and limb length equalization. While hip arthrodesis provides pain relief and joint stability, it has obvious drawbacks of eliminating hip motion and adverse effects on the contralateral hip and knee, and lower back $[4,5]$. THR has become the standard treatment method for hip diseases in the elderly. But, young patients may undergo two or more revision surgeries in their life because of limited lifetime of the joint prosthesis. So, THR is not the first choice for adolescent and young adults [1]. Moreover, THR of a deficient hip in adolescents and young adults is technically difficult, does not counteract the gluteal muscles insufficiency in a proper way, and fraught with significant complications as early postoperative dislocation, femoral nerve and peroneal nerve palsies, femoral shaft fracture, late infection, and aseptic loosening $[6,7]$.

Hosny G.A., Ahmed A.-S. A.-A. Is arthroplasty inevitable after Ilizarov hip reconstruction of unstable hip joints in adolescents and young adults? Long-Term Evaluation of 136 Cases. Genij Ortopedii, 2021, vol. 27, no 3, pp. 351-356. DOI 10.18019/1028-44272021-27-3-351-356 
Pelvic support osteotomy has a long tradition in orthopaedic surgery and trace back to the first half of the nineteenth century [8]. The aim is to reorient the biological tissues to improve gait and to offer pelvic support. Numerous variations have been described by several authors, most notably by Lorenz and Schanz [8-10]. The disadvantages of these early osteotomies were a significant additional leg-length discrepancy, and lack of correction of the mechanical axis resulting in malalignment with valgus angulation with secondary abnormal mechanical forces on the knee [11].

Gavriil Ilizarov was an accredited surgeon to develop the proximal femoral valgus osteotomy combined with a distal lengthening and varus osteotomy aiming to restore physiological loads on the knee and ankle joints and to correct leg length discrepancy (LLD). Moreover, he emphasized the importance of additional extension at the proximal osteotomy site. This procedure is called the Ilizarov hip reconstruction $[10,12,13]$.

Up to the authors' information, all available studies of Ilizarov pelvic support osteotomy present a shortterm to mid-term results of a small series of patients. The purpose of this study is to evaluate the long-term radiographic and clinical outcomes of this approach for the treatment of painful and unstable hips in adolescents and young adults.

\section{PATIENTS AND METHODS}

The inclusion criteria of this retrospective study were patients with painful and unstable hip joints of variable causes with treatment by Ilizarov pelvic support osteotomy. Cases with a follow-up of less than five years were excluded. The study included 136 cases with an average age of 18.3 (range, 6 to 34) years with 75 male patients $(55.1 \%)$ and 61 females $(44.9 \%)$. The primary causes of the hip instability were untreated or unsuccessfully treated cases of septic arthritis (40 cases; $29.4 \%$ ) (Fig. 1), congenital hip dislocation (28 cases; $20.6 \%$ ) (Fig. 2), paralytic hip dislocation (36 cases; $26.5 \%$ ), proximal femoral focal deficiency (14 cases; $10.3 \%)$, neglected fracture neck femur (10 cases; $7.4 \%$ ), osteoarthritis ( 6 cases; $4.4 \%$ ), and tuberculous hip arthritis ( 2 cases; $1.5 \%$ ).

The patients presented with hip pain, limping, instability, restricted hip ROM, and shortening. All patients used crutches or for ambulation particularly in outdoor. Assessment of patients started clinically by detailed history of the primary etiological problem and any previous treatment methods with the history of the current functional status and pain. This was followed by careful clinical examination of the ipsilateral hip, knee, and ankle joints, in addition to the contralateral joints and spine. Joint ROM, instability, Trendelenburg test (as described by Hardcastle and Nade [14]), LLD, associated flexion contracture, and detailed neurovascular assessment were all considered. LLD was evaluated clinically by the block test and radiographically by long film radiographs. Functional evaluation was done preoperatively and at the last follow-up using the Harris Hip Scoring System [15]. Harris hip score represents pain, walking function, daily living activities, and hip ROM.

The preoperative radiological assessment and planning is crucial in these complex hip disorders. This included evaluation of an anteroposterior (AP) view of the pelvis with imaging of the affected hip in maximum abduction view made in supine position while the lower extremities are adducted and the affected hip is flexed and adducted over the top of the uninvolved side. Additionally, a long film standing AP view of both lower limbs, and a single-limb stance AP view of each lower extremity were made.

All patients had to be seen by an arthroplasty surgeon to discuss the advantages and disadvantages of arthroplasty in the young age. The details of the procedure were explained to the patients or to their guardians with preoperative group meeting with similar cases in different stages of treatment or follow-up.

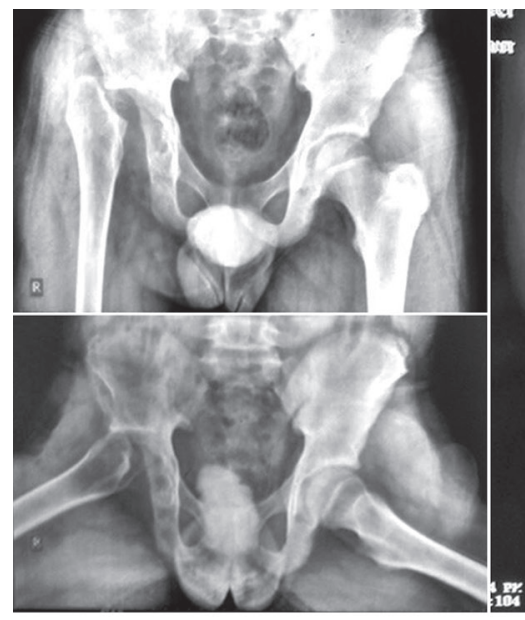

a

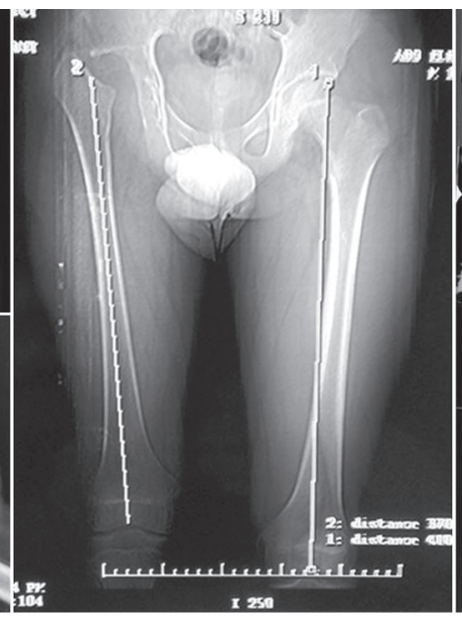

b

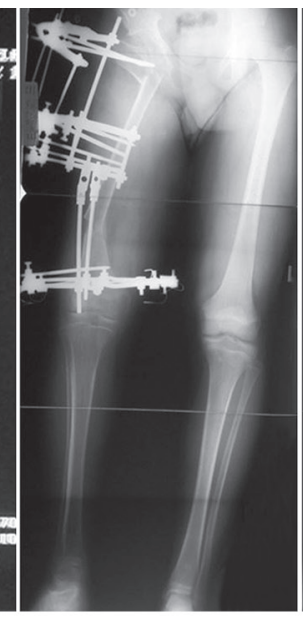

C

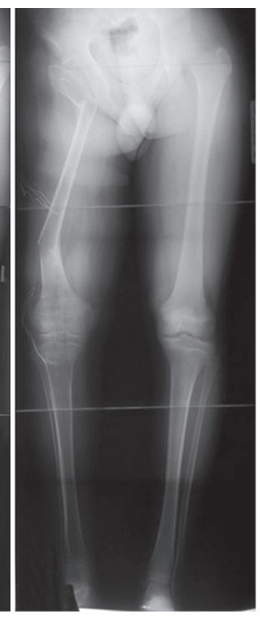

d

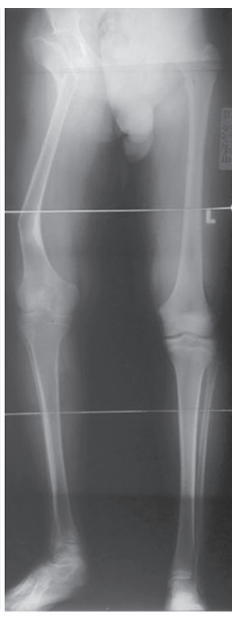

e

Fig. 1 (a) 16-year old boy, old septic left hip arthritis with absent head and neck; (b) CT scanogram of the patient; (c) X-ray before frame removal; $(\boldsymbol{d})$ X-Ray after frame removal; $(\boldsymbol{e})$ follow-up X-ray after one year 


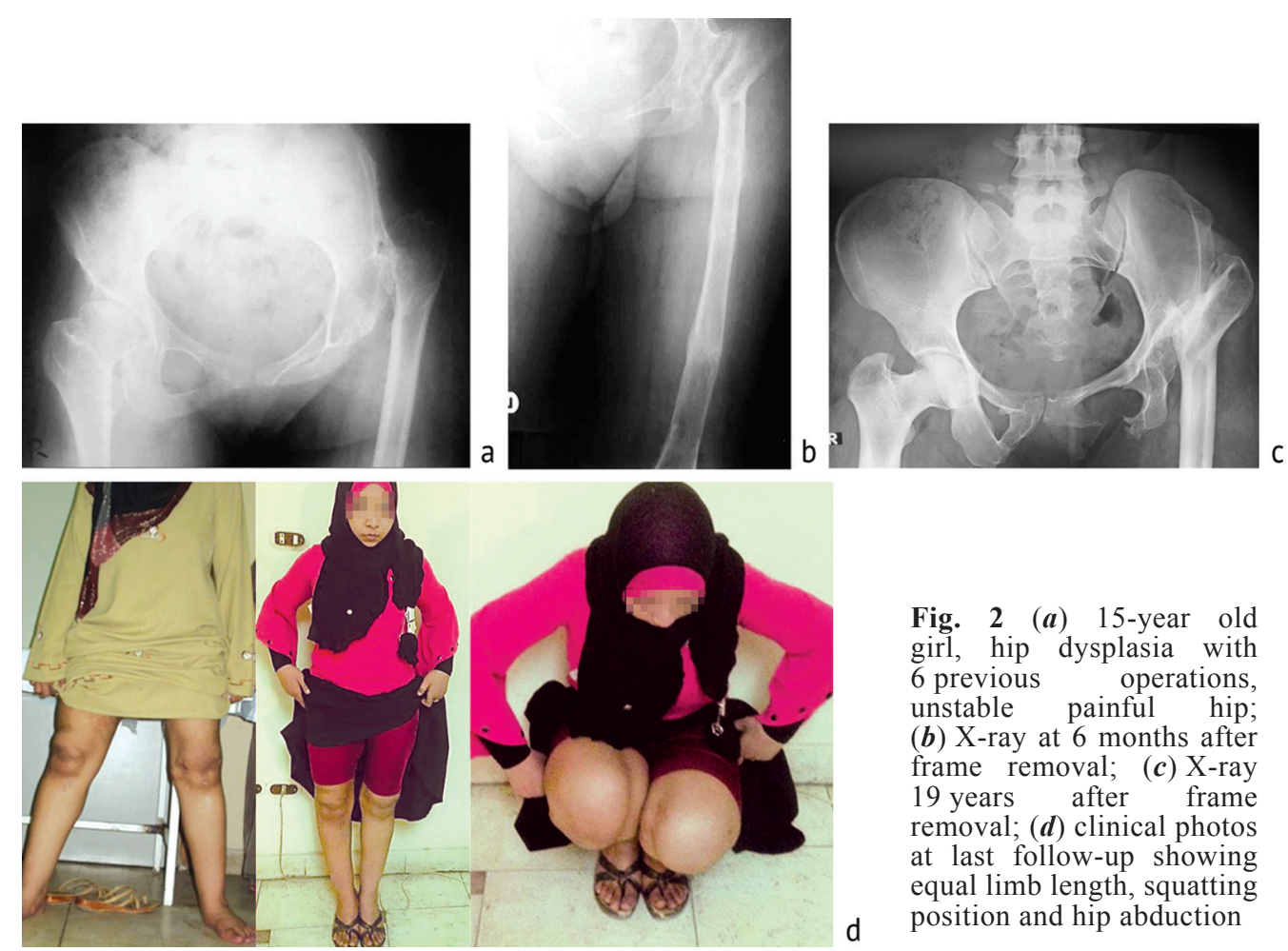

\section{Operative technique}

The patients were placed on in a supine position on a radiolucent table under general or epidural anesthesia according to the choice of the anesthesia consultant. The affected extremity and hemi-pelvis were draped free to permit circumferential access to the whole limb. The levels of the osteotomies were determined under image intensifier. The proximal valgus osteotomy was chosen at the site of abutment of the proximal femur with the pelvis in AP view with the hip in maximum adduction. In this position, the proximal femur is adducted and flexed. Owing to this position and secondary to the pathological anatomy, the femur externally rotates. This rotation must be taken into consideration [10]. Proximal valgus osteotomy was determined at the acetabular level and in others it was at the sub-acetabular level. The Ilizarov frame consisted of two femoral arches attached to the proximal femur. With full hip adduction, two or three 6-mm Schanz pins were inserted in the proximal femoral segment proximal and distal to the determined level of the proximal osteotomy and fixed to two arches. The proximal Schanz pins were mounted parallel to the horizontal axis of the pelvis. The arch was oriented with inclination in the sagittal plan in a degree corresponding to the degree of the preoperative hip flexion contracture. This proximal frame was planned for correcting valgus, extension, and internal rotation deformities. The middle femoral segment was anchored to one 5/8 ring with three Schanz pins inserted perpendicular to the mechanical axis of this segment. The distal femoral segment was attached to one ring or one ring with $5 / 8$ rings for using $1.8-\mathrm{mm}$ wires placing the ring parallel to the knee joint.

The proximal valgus osteotomy was done without violating the hip joint or excision of the femoral head. The level of the proximal osteotomy was acetabular in 22 cases $(16.2 \%)$ and sub-acetabular in 114 cases $(83.8 \%)$. The proximal osteotomy was done through a small lateral approach with an osteotome after multiple predrilling. The distal osteotomy was done in a similar way just distal to the junction between the middle and lower thirds of the femur.

The image intensifier was used to check completeness of osteotomies and alignment, and to ensure the proximal segment was locked into its proper position to support the hemi-pelvis. Placing the lateral and distal and cortices of the proximal segment into the medullary canal of the distal segment increases stability. Losing the contact between these segments will render the osteotomy site unstable. Generally, the arches and rings will be parallel at the end of the procedure. The limb rotation and the mechanical axis were evaluated. There was no overcorrection of the valgus angle achieved at the proximal osteotomy. Similarly, no overcorrection of extension was done to the amount of the predetermined flexion contracture. The limb segment distal to the proximal osteotomy level was kept in a way to be parallel to the contralateral healthy lower limb. The rotation correction through the proximal extension osteotomy was performed in such way to keep the patella facing forward. The degree of varus at the distal osteotomy was chosen aiming at keeping a straight mechanical axis of the lower limb from the point of contact of the proximal femur segment to the pelvis down to the knee and the ankle joint without any valgus malalignment. After wound closure and soft tissue release around the pins, a bulky compressive dressing is applied.

Postoperative care

Partial weight-b earing was encouraged as soon as tolerated postoperatively. Physical therapy with activeassisted and active ROM exercises started early to strengthen the abductors, extensors, and flexors of the hip and to maintain hip and knee ROM. The patients were discharged on the second or third postoperative day. Care of pin sites was explained to the patients or their guardians. Distal osteotomy distraction was started after a latency period of seven days and continued at a rate of 1 
mm per day. The distraction rate was modified depending on the regenerate quality in the follow-up radiographs. The follow-up visits were scheduled bi-weekly till achievement of the required correction of the mechanical axis and length. The patients were clinically evaluated for the condition of the wounds, stability of the frame, pin sites, LLD, ROM, and progression of weight-bearing. Equalization of the both limb lengths was evaluated by the block test and long film standing AP radiograph. Radiographic evaluation included adjustment of the mechanical axis, healing of the proximal osteotomy, and progression of the distal regenerate and its consolidation.

Thereafter, the patients were followed up every month till removal of the Ilizarov fixator. The frame was removed after radiographic evidence of healing with crossing trabeculae and absence of any radiolucent lines proximally and at least three cortices in orthogonal radiographs distally. Clinical evidence of healing was painless full weight-bearing with loose rods. The fixator was removed in the clinic without anesthesia. No protection braces or casts were applied. Then, intensive ROM and abductor muscle-strengthening exercises were continued for six months. After frame removal the patients were seen every two months for the first six months, and yearly thereafter with radiographic assessment and functional evaluation by Harris hip scores, Trendelenburg sign, and the patient's overall degree of satisfaction.

\section{RESULTS}

External fixation period ranged from 4 to 12 months (average, 6.5 months). The patients were followed up for a mean of 17.4 years (range, 5-27 years). Multiple clinical parameters showed significant improvement at the final follow-up including pain, pain-free walking distance, limping, hip flexion, hip abduction, hip flexion contracture, lumbar lordosis, and LLD. Functionally, the mean Harris hip score improved with a statistically significant difference from 48 (range, 35-65) preoperatively to 83 (range, 70-90) postoperatively. Pain disappeared in all patients except six cases in the early postoperative period. Four of them showed improvement with physiotherapy over time. The need for the previously used walking aids was obviated in all cases except in the two cases with persistent exertional pain. Walking ability and pain-free walking distance improved in all patients from an average of $35 \mathrm{~m}$ (range, 10 to 50) preoperatively to $1150 \mathrm{~m}$ (range, 1000 to $1500 \mathrm{~m}$ ) postoperatively with a statistically significant difference.

Trendelenburg gait disappeared in 106 patients $(77.9 \%)$. The primary pathology of the remaining 30 cases ( $22.1 \%)$ was paralytic hip dislocation (15 cases), high DDH (six cases), and late sequelae of septic arthritis (nine cases). The achieved lengthening ranged from 3.5 to $12 \mathrm{~cm}$ with an average of $6.4 \mathrm{~cm}$. The residual LLD averaged $0.8 \mathrm{~cm}$. The mean radiology consolidation index for callus distraction was 1.4 months $/ \mathrm{cm}$ (range, 1-2.5 months $/ \mathrm{cm}$ ). The mean Ilizarov frame index was 1.7 months $/ \mathrm{cm}$ (range, 1.5-2.6).
The final mean hip flexion ROM showed a statistically significant improvement postoperatively into $120^{\circ}$ (range, $100-130^{\circ}$ ) compared to $80^{\circ}$ (range, $70-110^{\circ}$ ) preoperatively. Additionally, the average hip abduction ROM improved from $8^{\circ}$ (range, $0-10^{\circ}$ ) preoperatively to $20^{\circ}$ (range, $10-35^{\circ}$ ) postoperatively with a statistically significant difference. The mean achieved valgus angulation at the end of the follow-up period was $41^{\circ}$ (range, $30-50^{\circ}$ ), and the average extension angulation was $14^{\circ}$ (range, $10-20^{\circ}$ ). Distally, the mean varus angulation was $30^{\circ}$ (range, $20-38^{\circ}$ ).

At the last follow-up visit at a mean of 17.4 years, all patients were satisfied with the procedure except one. According to the ROM, pain, activities, and the functional score none of the patient required total hip arthroplasty.

Complications included some sort of superficial pin tract infection in all cases. This was treated by oral antibiotics and careful pin site dressing. No pin or wire demonstrated loosening that required removal or replacement. Fracture of the regenerate occurred in 3 cases following a fall. These fractures were treated successfully by cast application. All cases showed some degree of reduced knee range of flexion immediately after frame removal. However, this was markedly improved by intensive and regular physiotherapy. Knee stiffness persisted in 3 cases. These cases were treated by soft tissue release. Progressive loss of angulation at the subtrochanteric level developed in 3 cases.

\section{DISCUSSION}

Being a multi-factorial complex problem, unstable hip joint in adolescents or young adults requires a multipurpose solution. Arthrodesis provides stability but eliminates motion and has known drawbacks on the spine and other joints. Whereas THR restores motion, it is technically difficult in these complex situations, and is associated with several complications and high failure rate. Moreover, the possible high revision rate remains a concern in adolescents and young adults $[1,4,5]$. Girard et al. [16] studied the risk factors for revision of hip arthroplasties and found that revisions are likely in patients younger than 30 years, with a high complication rate and low survivorship of the hip revision. They recommended alternative solutions whenever possible.

Ilizarov pelvic support osteotomy provides several roles in treatment of challenging hip disorders including biomechanical, functional, and aesthetic aspects. Biomechanically, this approach provides hip stability while preserving motion through increasing the support surface between the femur and pelvis. Also, it creates a stable fulcrum with consequent diminished abductor torque needed to gain pelvic equilibrium by shifting the fulcrum point medially. The valgus osteotomy tightens the gluteus medius by increasing the distance between the pelvis and the greater trochanter $[6,11]$. The study of Inan et al. [6] demonstrated that pelvic support osteotomy was effective in restoration of abductor muscle length and volume. These several effects could attribute to correction of the Trendelenburg limp. Additionally, this technique allows adjustment of the mechanical axis, equalization of the limb lengths, and reduces lumbar lordosis [11]. 
Functionally, this method of hip reconstruction keeps hip motion, alleviates pain, and improves gait and daily activities. Several studies demonstrated significant improvement in the functional scores $[2,5-8,10,17,18]$. The aesthetic issues include improved personal hygiene and sexual activity with greater hip abduction. Furthermore, the thigh soft tissues mask the femoral angulation and the proximal valgus of the femur moves the gluteal region laterally giving a better appearance aspect compared to the preoperative look of hypotonic hip [11].

Ilizarov circular fixator is versatile offering threedimensional correction, adequate stability permitting early ROM, and feasibility of postoperative finetuning or adjustment of the mechanical axis and length. However, the bulk of the frame could be cumbersome to the patient. Trying to avoid this disadvantage, some authors used monolateral external fixators [5], others reported internal fixation modalities, and others used multiple methods in the same study [17]. However, these are either small case series [19] or isolated case reports $[9,20,21]$ with occasional two-stage procedures or short-term follow-up. Whereas mono-lateral external fixators are much more comfortable to patients, they are biomechanically less resistant to the shearing forces [17]. Inan and Bowen [5] used this approach to treat 16 patients with a mean age of 25.3 years using monolateral external fixator. The mean external fixation period was 7 months. After an average follow-up of 52.5 months, the mean Harris hip score increased from 50 preoperatively to 87.6 points postoperatively. Trendelenburg limp persisted in four cases. One patient suffered delayed consolidation, fracture at the distal osteotomy site, LLD of $3 \mathrm{~cm}$, and knee stiffness.

Krieg et al. [9] reported one case with fixation of the proximal osteotomy by a locking compression plate and a motorized intramedullary distraction nail for the distal lengthening. However, the follow-up was only 12 months and the authors stated that the technique is restricted by the geometric preconditions. Another case was reported by Bytyqi et al [20]. Surgery was done in two stages. The first was 6-cm femoral lengthening with a monolateral fixator. The second consisted of pelvic support osteotomy fixed by a special angulated plate and 4-cm distal lengthening with a monolateral fixator.

Metikala et al. [19] treated eight cases by two-stage reconstruction. The first stage included resecting the femoral head and pelvic support osteotomy fixed by double plating, and the second was distal femoral osteotomy for lengthening by a retrograde magnetic nail with a mean follow-up of 19 months. All osteotomies healed with a bone healing index of 47 days $/ \mathrm{cm}$. Trendelenburg sign disappeared in three cases. One patient suffered failure, and another had a $3.5-\mathrm{cm}$ lateral mechanical axis deviation needing osteotomy. However, in this study, the distal varus was not addressed with this approach, and, in contrast to using the Ilizarov fixator, the patients in this study were prevented from early weight-bearing. Moreover, the minimally invasive technique used for osteotomy with the Ilizarov fixator is more biologically sound compared to the used approach with double plate fixation. Unlike Ilizarov fixator, internal fixation techniques do not allow any postoperative corrections. Additionally, the high cost is a consideration with the internal lengthening nails $[19,22]$.

In contrast to Metikala et al. [19], we did not resect the femoral head in the current series but preserved the hip joint keeping the minimally invasive small wounds for osteotomy without further dissection. Leaving the femoral head or its stump did not have any negative effects on the outcomes of our patients. Femoral head resection was recommended by Milch [23] for gaining hip mobility and relieve of pain. Some authors reported femoral head resection $[6,19,24]$. Like the current study, Schiltenwolf et al. [25] reported satisfactory outcomes in 24 cases of congenital hip dysplasia treated by subtrochanteric valgus osteotomy without resecting the femoral head. Similarly, the femoral head was kept in the recent study of Luo et al [1].

In the present study, there was no attempt to add overcorrection to the proximal femoral valgus. Consequent drawbacks were not observed apart from progressive loss of angulation secondary to remodeling in three cases $(2.2 \%)$. Milch reported that whereas overcorrection of proximal femoral valgus leads to better stability of the hip, it is not recommended because it results in a decrease in the adduction range [17, 26]. Several authors recommended overcorrection of this valgus osteotomy $[4,8,17,18,19,27]$. However, this overcorrection is entirely empirical in prediction of osteotomy site remodeling and atrophy of the interposed soft tissues between the pelvis and the proximal femur $[4,27]$. Inan et al. [6] reported this remodeling in one of 11 patients. Rozbruch et al [10] reported this problem in two of eight cases. Gürsu et al. [17] observed correction losses in two of 20 patients. Despite few reports of remodeling, some authors considered an age younger than 12 years as a relative contraindication [3]. Regarding limb length equalization, over-lengthening should be avoided because it is not tolerated in the hip which is already in full adduction [4].

At the last follow up, all patients except one were fully satisfied with their outcomes and none was indicated for hip arthroplasty. THR following pelvic support osteotomy could be a challenging task due to the anatomical changes caused by proximal femoral angulation in both frontal and sagittal planes. THR was reported to treat a 29-year old woman after 15 years of previous pelvic support osteotomy. However, revision of the femoral component was done due to penetration of the proximal femur. The authors did not present how long the follow-up was and whether it was eventful or not [28].

\section{CONCLUSIONS}

Ilizarov pelvic support osteotomy provided a multipurpose solution to the complex challenging problem of hip instability in adolescents and young adults with variable primary etiologies. The improvements in the hip motion, mechanical axis, and correction of limb-length discrepancy lead to good functional outcomes over a long-term followup. This treatment modality might avoid or postpone the need for total hip arthroplasty for several years. 


\section{REFERENCES}

1. Luo S., Kong L., Wang J., Nie H., Luan B., Li G. Development of modified Ilizarov hip reconstruction surgery for hip dysfunction treatment in adolescent and young adults. J. Orthop. Translat., 2020, vol. 27, pp. 90-95. DOI: 10.1016/j.jot.2020.11.002

2. El-Mowafi H. Outcome of pelvic support osteotomy with the Ilizarov method in the treatment of the unstable hip joint. Acta Orthop. Belg., 2005, vol. 71, no. 6, pp. 686-691.

3. Umer M., Quadri T.A., Rashid R.H. Ilizarov hip reconstruction osteotomy - A review. Int. J. Surg., 2018, vol. 54, Pt. B, pp. 351-355. DOI: 10.1016/j. ijsu.2017.08.561

4. Pafilas D., Nayagam S. The pelvic support osteotomy: indications and preoperative planning. Strategies Trauma Limb Reconstr., 2008, vol. 3, no. 2, pp. 83-92. DOI: 10.1007/s11751-008-0039-7

5. Inan M., Bowen R.J. A pelvic support osteotomy and femoral lengthening with monolateral fixator. Clin. Orthop. Relat. Res., 2005, vol. 440, pp. 192-198. DOI: 10.1097/01.blo.0000180602.00487.47

6. Inan M., Alkan A., Harma A., Ertem K. Evaluation of the gluteus medius muscle after a pelvic support osteotomy to treat congenital dislocation of the hip. J. Bone Joint Surg. Am., 2005, vol. 87, no. 10, pp. 2246-2252. DOI: 10.2106/JBJS.D.02727

7. Mahran M.A., Elgebeily M.A., Ghaly N.A., Thakeb M.F., Hefny H.M. Pelvic support osteotomy by Ilizarov's concept: Is it a valuable option in managing neglected hip problems in adolescents and young adults? Strategies Trauma Limb Reconstr., 2011, vol. 6, no. 1, pp. 13-20. DOI: 10.1007/ s11751-011-0104-5

8. Mandar A., Tong X.B., Song S.H., Park Y.E., Hong J.H., Lee H., Song H.R. Pelvic support osteotomy for unstable hips using hybrid external fixator: case series and review of literature. J. Orthop. Sci., 2012, vol. 17, no. 1, pp. 9-17. DOI: 10.1007/s00776-011-0173-9

9. Krieg A.H., Lenze U., Hasler C.C. Ilizarov hip reconstruction without external fixation: a new technique. J. Child. Orthop., 2010, vol. 4, no. 3, pp. 259-266. DOI: $10.1007 / \mathrm{s} 11832-010-0256-8$

10. Rozbruch S.R., Paley D., Bhave A., Herzenberg J.E. Ilizarov hip reconstruction for the late sequelae of infantile hip infection. J. Bone Joint Surg. Am., 2005, vol. 87, no. 5, pp. 1007-1018. DOI: 10.2106/JBJS.C.00713

11. Catagni M., Guerreschi F., Lovisetti L., Tsibidakis H.. Pelvic support osteotomy (PSO): Indications, limits and complications. Kocaoğlu M., Tsuchiya H., Eralp L., eds. Advanced Techniques in Limb Reconstruction Surgery. Heidelberg, Springer, 2015, pp. 433-459. DOI: 10.1007/978-3642-55026-3 21

12. Ilizarov G.A. Hip dislocations. Green S.A., editor. Transosseous osteosynthesis. Theoretical and clinical aspects of regeneration and growth of tissue. Berlin, Heidelberg, Springer-Verlag, 1992, pp. 701-726. DOI: 10.1007/978-3-642-84388-4

13. Ilizarov G.A., Samchukov M.L. Rekonstruktsiia bedrennoi kosti po Ilizarovu v lechenii deformiruiushchego artroza tazobedrennogo sustava [Reconstruction of the femur by the Ilizarov method in the treatment of arthrosis deformans of the hip joint]. Ortop. Travmatol. Protez., 1988, no. 6, pp. 10-13. (in Russian)

14. Hardcastle P., Nade S. The significance of the Trendelenburg test. J. Bone Joint Surg. Br., 1985, vol. 67, no. 5, pp. 741-746. DOI: 10.1302/0301-6 20X.67B5.4055873

15. Harris W.H. Traumatic arthritis of the hip after dislocation and acetabular fractures: treatment by mold arthroplasty. An end-result study using a new method of result evaluation. J. Bone Joint Surg. Am., 1969, vol. 51, no. 4, pp. 737-755.

16. Girard J., Glorion C., Bonnomet F., Fron D., Migaud H. Risk factors for revision of hip arthroplasties in patients younger than 30 years. Clin. Orthop. Relat. Res., 2011, vol. 469, no. 4, pp. 1141-1147. DOI: 10.1007/s11999-010-1669-x

17. Gürsu S., Demir B., Yildirim T., Er T., Bursali A., Sahin V. An effective treatment for hip instabilities: pelvic support osteotomy and femoral lengthening. Acta Orthop. Traumatol. Turc., 2011, vol. 45, no. 6, pp. 437-445. DOI: 10.3944/AOTT.2011.2323

18. Marimuthu K., Joshi N., Sharma C.S., Bhargava R., Meena D.S., Bansiwal R.C., Govindasamy R. Ilizarov hip reconstruction in skeletally mature young patients with chronic unstable hip joints. Arch. Orthop. Trauma Surg., 2011, vol. 131, no. 12, pp. 1631-1637. DOI: 10.1007/s00402-0111376-4

19. Metikala S., Kurian B.T., Madan S.S., Fernandes J.A. Pelvic Support Hip Reconstruction with Internal Devices: An Alternative to Ilizarov Hip Reconstruction. Strategies Trauma Limb Reconstr., 2020, vol. 15, no. 1, pp. 34-40. DOI: 10.5005/jp-journals-10080-1450

20. Bytyqi C., Morina F., Salihaj N., Qorraj H., Bytyqi D., Shabani B. The Pelvic Support Osteotomy After Type IVA Septic Arthritis of the Hip. Med. Arch., 2014, vol. 68, no. 6, pp. 422-423. DOI: 10.5455/medarh.2014.68.422-423

21. Reynders-Frederix P., Reynders-Frederix C., Wajid M. Pelvic Support Osteotomy; Salvage Procedure in Chronically Dislocated Hips Case Report: Technical Note on the On-lay Bone Positioning of a Lengthening Nail and Review of the Literature. Open Orthop. J., 2016, vol. 10, pp. 232-240. DOI: $10.2174 / 1874325001610010232$

22. Alrabai H.M., Gesheff M.G., Conway J.D. Use of internal lengthening nails in post-traumatic sequelae. Int. Orthop. 2017. Vol. 41, No 9. P. 19151923. DOI: $10.1007 / \mathrm{s} 00264-017-3466-6$

23. Milch H. The resection-angulation operation for hip-joint disabilities. J. Bone Joint Surg. Am., 1955, vol. 37-A, no. 4, pp. 699-717.

24. Pontes M.D.S., Bortolin P.H., Volpon J.B. Double femoral osteotomy for the treatment of severe sequelae of the immature hip. Acta Ortop. Bras., 2020, vol. 28 , no. 6, pp. 287-290. DOI: 10.1590/1413-785220202806234170

25. Schiltenwolf M., Carstens C., Bernd L., Lukoschek M. Late results after subtrochanteric angulation osteotomy in young patients. J. Pediatr. Orthop. B, 1996, vol. 5, no. 4, pp. 259-267.

26. Milch H. The postosteotomy angle. J. Bone Joint Surg. Am., 1944, vol. 26, pp. 394-400.

27. Choi I.H., Cho T.J., Yoo W.J., Shin C.H. Recurrent dislocations and complete necrosis: the role of pelvic support osteotomy. J. Pediatr. Orthop., 2013, vol. 33, no. Suppl. 1, pp. S45-S55. DOI: 10.1097/BPO.0b013e318281216b

28. Thabet A.M., Catagni M.A., Guerreschi F. Total hip replacement fifteen years after pelvic support osteotomy (PSO): a case report and review of the literature. Musculoskelet. Surg., 2012, vol. 96, no. 2, pp. 141-147. DOI: 10.1007/s12306-011-0178-8

Received: 31.03 .2021

\section{Information about the authors:}

1. Gamal Ahmed Hosny, M.D., Professor,

Benha University, Faculty of Medicine, Egypt,

Email: gamalahosny@yahoo.com

2. Abdel-Salam Abdel-Aleem Ahmed, M.D.,

Benha University, Faculty of Medicine, Egypt,

Email: salam_ilizarov@yahoo.com 